\title{
Pneumonia complicada por pneumatocele gigante em criança com síndrome da imunodeficência adquirida: importância da fisioterapia respiratória
}

Pneumonia complicated by a giant pneumatocele in a child with acquired immunodeficiency syndrome: importance of chest physiotherapy

Silvia Regina M. de Paula', Melissa Pinto Gurgel', Luciana Macchiaverni ${ }^{3}$ Fabíola Meister Pereira ${ }^{4}$, Maria Ângela G. de O. Ribeiro ${ }^{5}$, Camila Isabel S. Santos ${ }^{6}$

\section{RESUMO}

Objetivo: Descrever os efeitos do tratamento fisioterapêutico na prevenção de complicações respiratórias de uma criança com síndrome da imunodeficiência adquirida, que apresentava pneumatocele gigante.

Descrição do caso: Criança com oito anos de idade, do sexo feminino, encaminhada para acompanhamento fisioterapêutico em hospital universitário devido a quadro de hipersecreção pulmonar, histórico de pneumonias e presença de pneumatocele gigante. A conduta fisioterapêutica incluiu técnicas de higiene brônquica ativa, envolvendo fluxo aéreo expiratório, com o intuito de promover a desobstrução pulmonar sem causar risco de complicações à criança. O seguimento foi multiprofissional e direcionado à prevenção de infecções recorrentes e de ruptura da bolha, que tornariam necessária a internação hospitalar. Durante o acompanhamento, o quadro pulmonar manteve-se estável, sem aumento ou ruptura da pneumatocele, radiograficamente visível.

Comentários: A atuação da fisioterapia respiratória nos casos de pneumatocele gigante não está definida na literatura. Não há, até o momento, evidências de seus efeitos benéficos, bem como diretrizes a respeito dos métodos mais eficientes e seguros. Ao contrário, muito se discute sobre os riscos e complicações decorrentes dessa intervenção. No presente caso, a assistência fisioterapêutica regular foi importante para garantir a estabilidade do quadro pulmonar da criança, sendo necessários estudos com desenho metodológico apropriado para verificação de eficácia clínica.

Palavras-chaves: Pediatria; pneumatocele; pneumonia; infecções por HIV.

\section{ABSTRACT}

Objective: To describe the effects of chest physiotherapy in preventing respiratory complications in a child with acquired immunodeficiency syndrome and a giant pneumatocele.

Case description: An eight-year-old female child was sent to the physiotherapy service of a university hospital due to pulmonary hypersecretion, repeated pneumonias and presence of a giant pneumatocele. The physiotherapy team selected active airway clearence techniques and expiratory flow techniques to promote pulmonary hygiene and to reduce risks for respiratory complications. The follow-up around was accomplished by a multiprofissional team with
Instituição: Departamento de Pediatria da Faculdade de Ciências Médicas da Universidade Estadual de Campinas (Unicamp), Campinas, SP, Brasil ${ }^{1}$ Mestre; Supervisora dos Cursos de Especialização e Aprimoramento em Fisioterapia Pediátrica da FCM-Unicamp, Campinas, SP, Brasil

${ }^{2}$ Pós-graduada em Fisioterapia Pediátrica pelo Curso de Especialização da Escola de Extensão-Unicamp, Campinas, SP, Brasil

${ }^{3}$ Pós-graduada em Fisioterapia Pediátrica pelo Curso de Especialização da Escola de Extensão-Unicamp, Campinas, SP, Brasil

${ }^{4}$ Pós-graduada em Fisioterapia Pediátrica pelo Curso de Aprimoramento em Fisioterapia Pediátrica da FCM-Unicamp, Campinas, SP, Brasil ${ }^{5}$ Doutora; Coordenadora dos Cursos de Aprimoramento e Especialização em Fisioterapia Pediátrica da FCM-Unicamp, Campinas, SP, Brasil ${ }^{6}$ Doutora; Professora de Fisioterapia em Pediatria da Universidade do Estado de Santa Catarina, Florianópolis, SC, Brasil
Endereço para correspondência:

Camila Isabel da Silva Santos

Rua Lauro Linhares, 1371, apto 01/Ed. Luiz Gustavo

CEP 88036-002 - Trindade/SC

E-mail: cacaiss@yahoo.com.br

Conflitos de interesse: nada a declarar

Recebido em: 8/4/09

Aprovado em: 28/6/09 
the objective of avoiding recurrent pulmonary infections, pneumatocele's rupture and need for long-term hospitalization. The child's pulmonary condition remained stable and no changes in the size of the pneumatocele were seen in repeated X-rays.

Comments: The role of chest physiotherapy in patients with giant pneumatoceles is not well defined in the literature. There is no evidence of beneficial effects of this therapy and there are no guidelines that address the most efficient and safest techniques. Currently, much is discussed about the risks and complications arising from chest physiotherapy. In this case, regular physiotherapy intervention was important to maintain child's pulmonary integrity, but studies are needed to evaluate techiques and clinical relevant effects.

Key-words: Pediatrics; pneumatocele; pneumonia; HIV infections.

\section{Introdução}

A identificação, em 1981, da síndrome da imunodeficiência adquirida, habitualmente conhecida como Aids, tornou-se um marco na história da humanidade. Com o aumento da incidência dos casos de Aids em mulheres, desde o início da epidemia, principalmente nos países em desenvolvimento, observou-se elevação de crianças infectadas pelo vírus $\mathrm{HIV}^{(1)}$.

Na população pediátrica, a principal forma de transmissão é a vertical ou perinatal, caracterizada pela passagem do vírus da mãe infectada para o filho durante a gestação, parto ou amamentação ${ }^{(2)}$. Crianças infectadas diferem de adultos infectados em vários aspectos. Primeiramente, a doença progride mais rapidamente em crianças; a carga viral costuma ser maior do que a registrada em adultos; as infecções bacterianas invasivas são recorrentes e as infecções oportunistas apresentam-se como doenças primárias, com maior agressividade. Apesar dessas diferenças, as respostas celulares da criança ao HIV são similares às do adulto, com boa resposta ao regime agressivo de coquetéis de drogas antirretrovirais ${ }^{(3)}$.

A apresentação clínica inicial da infecção pelo HIV em crianças tem um amplo espectro de manifestações. Alguns pacientes apresentam imunodeficiência grave, enquanto outros têm achados inespecíficos como hepatoesplenomegalia, parotidite, atraso do crescimento, febre persistente e diarreia crônica. A encefalopatia progressiva apresenta-se como a primeira manifestação da infecção pelo HIV em 10 a $15 \%$ das crianças ${ }^{(1)}$.
A doença pulmonar e o progressivo acometimento respiratório contribuem de modo significativo para a morbidade e mortalidade dos pacientes pediátricos infectados pelo HIV. Cerca de $80 \%$ das crianças infectadas desenvolvem doença pulmonar e 10 a $15 \%$ delas evoluem com imunodepressão precoce e deterioração clínica nos primeiros dois anos de vida $^{(2)}$. As alterações imunológicas secundárias à infecção contribuem para que a pneumonia ocorra com maior frequência e gravidade.

Pneumatoceles são complicações frequentes em pneumonias graves, geralmente por Staphyloccocus aureus. Trata-se de distensão alveolar que leva ao aparecimento de cistos no parênquima pulmonar. Podem ser simplesmente lesões enfisematosas, mas, em sua maioria, são múltiplas cavidades císticas com paredes finas e acúmulo de $\mathrm{ar}^{(4)}$. Geralmente regridem espontaneamente com a melhora do quadro pneumônico, no entanto, requerem intervenção cirúrgica quando se tornam infectadas ou rompem para a cavidade pleural ${ }^{(5)}$.

A atuação da fisioterapia respiratória na pneumatocele, seja ela gigante ou não, tem sido pouco abordada na literatura. É consenso a necessidade de estabilização do quadro respiratório dos pacientes com essa afecção devido à possibilidade de formação de abscessos e pneumotórax. No entanto, o risco de rompimento da bolha durante a aplicação de algumas técnicas de fisioterapia respiratória, como a tapotagem e as pressões manuais, provoca relativa insegurança para sua indicação, principalmente na fase aguda da doença.

Pacientes na faixa etária pediátrica que apresentam, além da pneumatocele, hipersecreção pulmonar e incompetência imunológica são considerados grupos de risco para quadros infecciosos, pneumonias e exacerbações recorrentes. Para eles, a fisioterapia tem sido indicada; contudo, a relação risco-benefício do procedimento ainda é foco de discussão na maioria dos serviços de saúde e hospitais do Brasil e do mundo. Assim, o presente relato aborda essa temática clínica com o intuito de apresentar os efeitos benéficos de técnicas de fisioterapia respiratória, selecionadas criteriosamente, de acordo com o quadro de paciente com imunideficiência e portadora de pneumatocele gigante.

\section{Relato de caso}

Criança do sexo feminino, nascida em 1994, caucasiana, encaminhada para seguimento no Hospital de Clínicas da Universidade Estadual de Campinas (Unicamp) com diagnóstico, no primeiro ano de vida, de síndrome da imunodeficiência adquirida. 
O tratamento multidisciplinar envolvia as especialidades médicas de imunologia, neurologia e cirurgia pediátrica. Aos dois anos de idade, devido à aparente encefalopatia evolutiva - suspeitada em razão de retardo cognitivo, apraxia motora global, dificuldade na fala e na compreensão da linguagem, além de coordenação motora e equilíbrio pouco desenvolvidos - foram incluídos os atendimentos de Fisioterapia, Psicologia e Fonoaudiologia da instituição. Além do quadro motor, o comprometimento pulmonar direcionou para o seguimento frequente da equipe de Pneumologia.

Durante o acompanhamento, a paciente apresentou pneumonia intersticial crônica inespecífica leve e focal (com ausência de fungos, parasitas ou inclusões virais), com 11 períodos de exacerbação pulmonar. As radiografias de tórax revelam imagens diversas de opacidade, sempre de forma heterogênea, em todo o hemitórax ou lobo pulmonar.

Em 1999, exames de imagem evidenciaram a formação de pneumatocele no hemitórax esquerdo com duas bolhas (Figuras 1 e 2) e, posteriormente, a união destas em uma única lesão cística, constituindo uma pneumatocele gigante

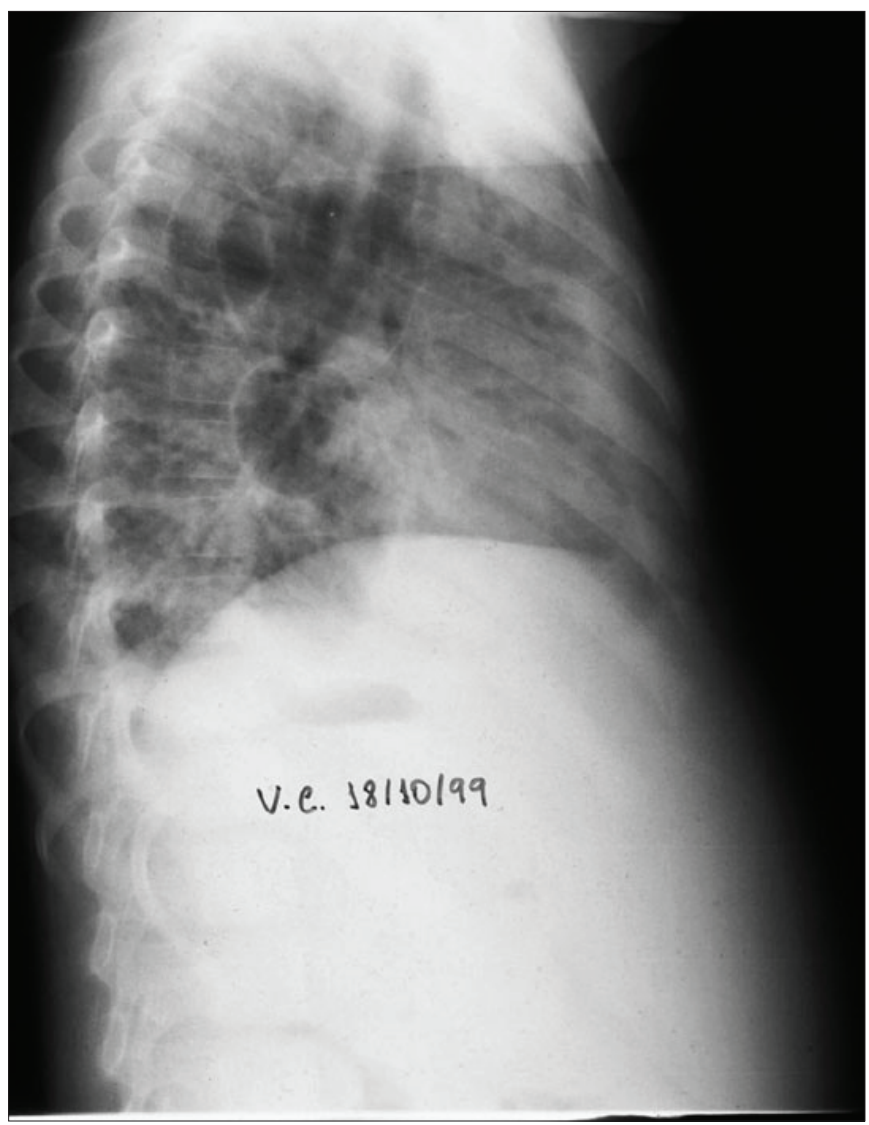

Figura 1 - Imagem hipertransparente com duas bolhas. A suspeita de pneumatocele levou à realização de tomografia de tórax
(Figura 3). Na tomografia de tórax de 1999 (Figura 2) já eram visíveis, além da pneumatocele, as imagens de carnificação em lobo médio direito, aumento da trama vascular e imagem em mosaico em lobo superior esquerdo.

A possibilidade de intervenção cirúrgica após a unificação das bolhas foi descartada pela equipe de Cirurgia Pediátrica devido ao quadro clínico instável da paciente. Adotou-se tratamento conservador, com antibioticoterapia e fisioterapia respiratória regular, sendo esta última mantida por quatro anos.

Diante da formação da pneumatocele gigante, o tratamento de fisioterapia respiratória passou a priorizar uma abordagem lúdica com enfoque na higiene brônquica e de vias aéreas superiores. Apesar da antibioticoterapia, a paciente apresentava grande quantidade de secreção pulmonar e episódios recorrentes de sinusopatia. Ao exame físico, a ausculta revelava murmúrio vesicular presente e diminuído à esquerda, com presença de estertores subcreptantes, creptantes e roncos difusos.

Diante da presença da pneumatocele gigante e da necessidade de manutenção da integridade pulmonar, as técnicas fisioterapêutica utilizadas ambulatorialmente incluíam: higiene nasal, desobstrução rinofaríngea retrógrada, vibração manual e mecânica, oscilação oral de alta frequência (OOAF), técnica de expiração forçada (TEF), ciclo ativo da respiração (CAR) e aumento do fluxo expiratório (AFE). A tapotagem associada à drenagem postural passou a ser introduzida na terapêutica após um ano de seguimento, diante da boa resposta da paciente à associação de técnicas e cronificação do quadro. A paciente comparecia duas a três vezes por semana

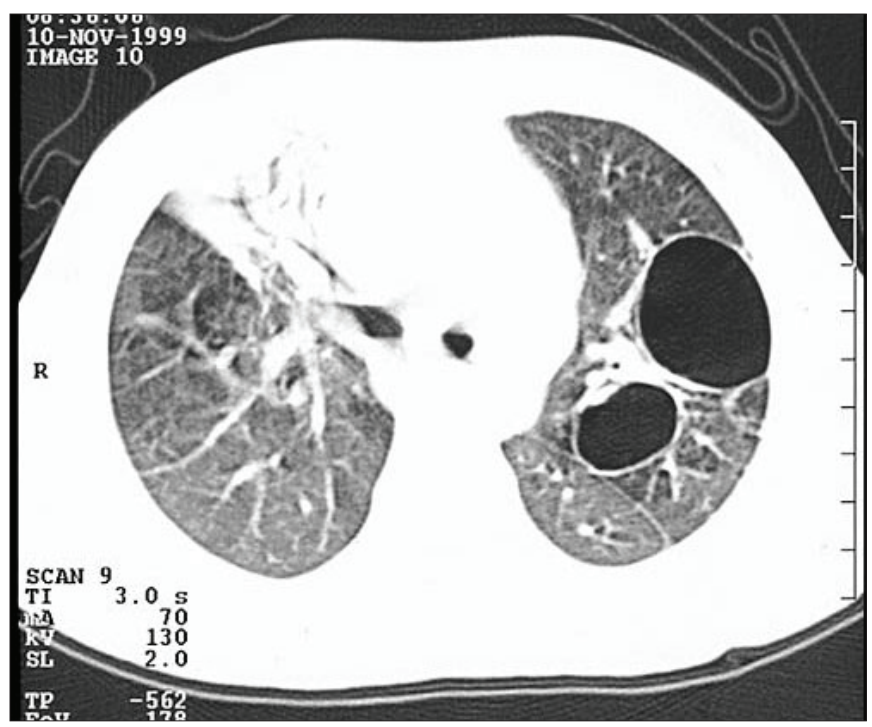

Figura 2 - Tomografia de tórax: carnificação em lobo médio direito, aumento da trama vascular e imagem em mosaico em lobo superior esquerdo 
para seguimento ambulatorial, totalizando aproximadamente 88 sessões em quatro anos.

Após a realização da fisioterapia, observava-se melhora imediata da ausculta pulmonar, com expectoração de secreção em grande quantidade e melhora da saturação periférica de oxigênio, que aumentava de 89 a $90 \%$ antes da terapia para $96 \%, \operatorname{logo}$ após a intervenção.

$\mathrm{O}$ tratamento regular realizado pelos fisioterapeutas garantiu a estabilização do quadro pulmonar e a prevenção de complicações, sendo esporádicos os episódios de piora respiratória e exacerbação pulmonar aguda, com baixa incidência de infecções bacterianas e redução do número de internações hospitalares (de seis internações por ano para apenas duas, em comparação às sofridas anteriormente, no início do acompanhamento fisioterapêutico). Durante as hospitalizações e nos atendimentos emergenciais realizados no pronto atendimento, deu-se continuidade à fisioterapia.

Embora apresentasse distúrbio cognitivo, a paciente colaborava ativamente e respondia bem aos comandos do terapeuta. A criança não teve bom desempenho apenas à realização da prova espirométrica.

\section{Discussão}

Na literatura, não são numerosos os relatos de casos de pneumatoceles em crianças, especialmente se portadoras de HIV. A incidência é alvo de controvérsias: alguns autores relatam ser uma complicação rara na infância ${ }^{(6)}$ e outros, um achado comum em crianças entre um e quatro anos de idade ${ }^{(5,7)}$.

$\mathrm{O}$ tratamento é geralmente conservador e com resolução espontânea, variando de semanas a vários meses. O tratamento cirúrgico é reservado para casos de pneumatocele com complicações, como pneumotórax e infecções ${ }^{(1,6,8)}$, quando a retirada da região acometida é indicada, principalmente se as lesões forem maiores de $10 \mathrm{~cm}^{(9)}$.

A atuação da fisioterapia na pneumatocele não é discutida na literatura, sendo contraindicada pela equipe médica, principalmente na fase aguda, logo que a imagem é detectada pelo exame radiológico. No entanto, conforme apresentado neste relato, a escolha por técnicas a fluxo pode ser uma alternativa para essa situação clínica. Considerando se tratarem de técnicas pouco agressivas aos pacientes pneumopatas crônicos ${ }^{(10)}$, quando bem indicadas, proporcionam a depuração mucociliar de forma segura e previnem a exacerbação e a piora do quadro respiratório.

Não há nenhum nível de evidência relatado na literatura que sustente a indicação de fisioterapia respiratória

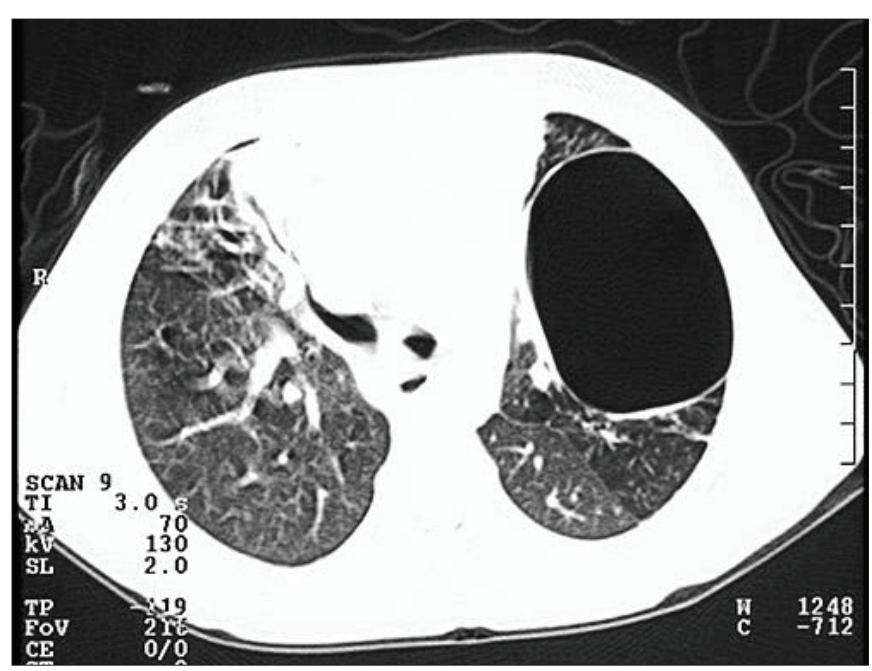

Figura 3 - Tomografia de tórax: união das bolhas em uma única lesão cística

na pneumatocele na fase aguda ou crônica. O que existem são resultados positivos na prática assistencial, como efeito fisioterapêutico imediato satisfatório (melhora da ausculta pulmonar, da saturação de oxigênio e do padrão respiratório), e conceitos gerados por discussões com especialistas e com equipe multiprofissional de alguns serviços.

O Departamento de Pneumologia da Sociedade de Pediatria de São Paulo, em 2002, indicou o uso de oxigenoterapia constante durante dez dias $(1 \mathrm{~L} / \mathrm{min})$, por meio de cateter nasal, como uma alternativa terapêutica para reduzir a bolha de pneumatocele aguda, com objetivo de reduzir o nitrogênio contido na cavidade. Assim, a equipe multiprofissional do Hospital de Clínicas da Unicamp procedeu conforme a recomendação, mas não houve redução da pneumatocele ou mudança no quadro radiológico. Optou-se, então, pela manutenção da antibioticoterapia e indicação da fisioterapia respiratória para evitar formação de abscesso pulmonar e ruptura da bolha, entre outras complicações comuns à pneumatocele. Nesse sentido, a condução do caso foi bem sucedida e não houve rompimento da bolha durante a fisioterapia e/ou instalação de processo infeccioso na cavidade. Vale ressaltar que não houve complicações do quadro e/ou intercorrências como hemoptise, queda da saturação, piora do desconforto ou ruptura da bolha durante a aplicação das técnicas selecionadas.

A indicação de fisioterapia na pneumatocele tem sido associada aos benefícios secundários à aplicação de técnicas de higiene brônquica, como adequação ventilatória e prevenção de infecções, já retratados na literatura. No entanto, o risco 
iminente de possíveis complicações decorrentes da aplicação de algumas técnicas fisioterapêuticas, sendo o pneumotórax a mais grave, indica avaliação minuciosa de cada caso específico para solicitação ou não dessa intervenção. Diante de pacientes crônicos, a necessidade de manutenção da permeabilidade da via aérea para evitar que outras complicações se instalem por exemplo, novas pneumonias, derrame pleural e formação de abscessos - fazem com que a fisioterapia respiratória seja considerada sempre que os benefícios se sobreponham aos riscos do procedimento.

Esse consenso parece estabelecido na prática clínica e, apesar da falta de evidências, discute-se a contraindicação absoluta da técnica de tapotagem na fase aguda, pelo risco de ruptura da bolha. Com relação às pressões exercidas durante outros procedimentos de fisioterapia, parece não haver risco de rompimento da bolha, considerando que a pressão gerada pela tosse é maior do que a gerada por qualquer outra técnica ou recurso terapêutico.

\section{Referências bibliográficas}

1. Cardoso CAA. Acompanhamento do curso da infecção pelo HIV em uma coorte de crianças e adolescentes atendidos em centro de referência em Belo Horizonte no período de 1989 a 2003 [tese de doutorado]. Belo Horizonte (MG): UFMG; 2006.

2. Souza PHVA. Função pulmonar de crianças e adolescentes infectados pelo vírus da imunodeficiência humana com pneumonia intersticial [tese de mestrado]. Campinas (SP): Unicamp; 2005.

3. Saloojee H, Violari A. HIV infection in children. BMJ 2001;323:70-4.

4. Quingley MJ, Frase RS. Pulmonary pneumatocele: pathology and pathogenesis. AJR 1988;150:1275-7.

5. Kunyoshi V, Cataneo DC, Cataneo AJM. Complicated pneumonias with empyema and/or pneumatocele in children. Pediatr Surg Int 2006;22: $186-90$.
Apesar do presente relato retratar o acompanhamento fisioterapêutico de um caso isolado de uma criança com pneumatocele gigante, a relevância da abordagem vai ao encontro da falta de material sobre o assunto. As evidências científicas são escassas e o registro de uma experiência assistencial no manejo dessa afecção pode ajudar na condução de quadros como este. Fica exposta, assim, a melhora respiratória imediata da paciente diante das condutas fisioterapêuticas adotadas, identificada pela ausculta pulmonar e promoção da higiene brônquica, bem como a manutenção da estabilidade do quadro pulmonar durante todo acompanhamento.

\section{Agradecimentos}

Os autores agradecem a Professora Doutora Adyléia Aparecida Dalbo Contrera Toro e ao Professor Doutor José Dirceu Ribeiro pelas contribuições clínicas e terapêuticas na condução do caso e sugestões científicas para elaboração deste manuscrito.

6. Holland ET, Saulsbury FT. Chronic Pneumocystis carinii pneumonia associated with extensive pneumatocele formation in a child with Human Immunodeficiency Virus infection. Pediatr Pulmonol 2003;35:144-6.

7. Tarpy SP, Komfeld H, Moroz K, Lazar HL. Unusual presentation of a large tension bronchogenic cyst in an adult. Thorax 1993;48:951-2.

8. İmamoğlua M, Çay A, Koşucu P, Özdemir O, Çobanoğlu U, Orhan F et al Pneumatoceles in postpneumonic empyema: an algorithmic approach. J Pediatr Surg 2005;40:1111-7.

9. Lattuada E, Puppini G, Faggian F, Lanzafame M, Concia E. Pneumatocele formation during Pneumocystis carinii pneumonia in a man with HIV-1 infection. Lancet 2004;4:170.

10. Schechter MS. Airway clearance applications in infants and children. Respir Care 2007;52:1382-91. 